\title{
Glucagon-like peptide-1 receptors in the brain: controlling food intake and body weight
}

\author{
Laurie L. Baggio and Daniel J. Drucker \\ Department of Medicine, Lunenfeld-Tanenbaum Research Institute, Mount Sinai Hospital, University of Toronto, Toronto, Ontario, Canada.
}

\begin{abstract}
The peptide hormone glucagon-like peptide-1 (GLP-1) enhances glucoseinduced insulin secretion and inhibits both gastric emptying and glucagon secretion. GLP-1 receptor (CLP-1R) agonists control glycemia via glucosedependent mechanisms of action and promote weight loss in obese and diabetic individuals. Nevertheless, the mechanisms and cellular targets transducing the weight loss effects remain unclear. Two recent studies in the $\mathrm{JCl}$ provide insight into the neurons responsible for this effect. Sisley et al. reveal that GLP-1R agonist-induced weight loss requires GLP-1Rs in the CNS while Secher et al. reveal that a small peptide GLP-1R agonist penetrates the brain and activates a subset of GLP-1R-expressing neurons in the arcuate nucleus to produce weight loss. Together, these two studies elucidate pathways that inform strategies coupling CLP-1R signaling to control of body weight in patients with diabetes or obesity.
\end{abstract}

\section{The metabolic effects of GLP-1}

Glucagon-like peptide-1 (GLP-1) is a small peptide hormone that is continuously secreted from enteroendocrine cells at a low level in the fasting or interprandial state. In response to nutrient ingestion, GLP-1 secretion is enhanced and circulating levels rise several-fold (1). The classical actions of GLP-1 include the ability to enhance glucose-stimulated insulin secretion; however, the known actions of GLP-1 were rapidly expanded to include the ability to inhibit gastric emptying and glucagon secretion. These combined actions fostered the development of multiple short- and long-acting GLP-1 receptor (GLP-1R) agonists for the treatment of type 2 diabetes. GLP-1R agonists also induce weight loss in patients with diabetes or obesity, further highlighting the potential for GLP-1R agonists to correct metabolic defects that arise in subjects with diabetes or obesity.
The physiological importance of endogenous GLP-1 in feeding behavior and energy homeostasis is illustrated by rodent studies showing that direct administration of the GLP-1R antagonist exendin(9-39) into the brain increases food intake in satiated rats (2). Conversely, knockdown of the proglucagon gene, which encodes GLP-1, in the nucleus of the solitary tract (NTS) promotes hyperphagia and weight gain in rats (3). Importantly, chronic peripheral antagonism of the GLP-1R for one week produced additional food intake and weight gain in high-fat diet-fed obese mice (4). In contrast, peripheral antagonism of the GLP-1R by intravenous infusion of exendin(9-39) for several hours did not significantly modulate food intake in human studies (5, 6). These discrepancies may reflect species-specific differences that include the relative accessibility and importance of GLP-1R circuits in the CNS compared with

Related Articles: pp. 2456, 4473

Conflict of interest: Daniel J. Drucker has served as an advisor or consultant within the past 12 months to Arisaph Pharmaceuticals Inc., Intarcia Therapeutics Inc., Merck Research Laboratories, Medlmmune, Novo Nordisk, NPS Pharmaceuticals Inc., Receptos Inc., Sanofi, Takeda, and Transition Therapeutics Inc. Daniel J. Drucker receives support for preclinical studies through grants to Mount Sinai Hospital from Merck \& Co., GlaxoSmithKline, Novo Nordisk, and Sanofi. Reference information: J Clin Invest. 2014;124(10):4223-4226. doi:10.1172/JCI78371.

those in the peripheral nervous system for controlling appetite, or these discrepancies may be due to other limitations, including the dose, potency, pharmacokinetics, and selectivity of the antagonist used and the short-term observation period.

\section{Complex GLP-1-dependent pathways and effects}

Attribution of the precise mechanisms that link GLP-1R signaling to body weight regulation in different species is challenging due to the complexity of pathways that regulate food intake and energy expenditure. The experiments associated with the original observations that GLP-1 inhibits food intake and produces satiety in rats used intracerebroventricular injections for delivery of GLP-1, a common paradigm for assessment of anorectic peptides (7). Subsequent studies demonstrated that centrally administered GLP-1 also induces an aversive response and activates sympathetic nervous system pathways, potentially indirectly controlling brown adipose tissue thermogenesis (8). The finding that GLP-1R agonists potently inhibited gastric emptying, which may also contribute to induction of satiety, further complicated interpretation of the GLP-1-dependant mechanisms leading to weight loss. Although nausea and vomiting, which may reflect an aversive response or reduced gastric emptying, are common side effects associated with GLP-1R agonist therapy, these complaints are transient, and many subjects experience weight loss without ever reporting these symptoms. Furthermore, GLP-1R-dependent inhibition of gastric emptying is subject to rapid tachyphylaxis $(9,10)$, and long-acting GLP-1R agonists or continuous administration of native GLP-1 produce weight loss independent of changes in gastric emptying (9).

The relative contributions of signals from regions within the peripheral nervous system or CNS that transduce the anorectic effects of GLP-1R agonists are not known precisely; however, candidates include the 


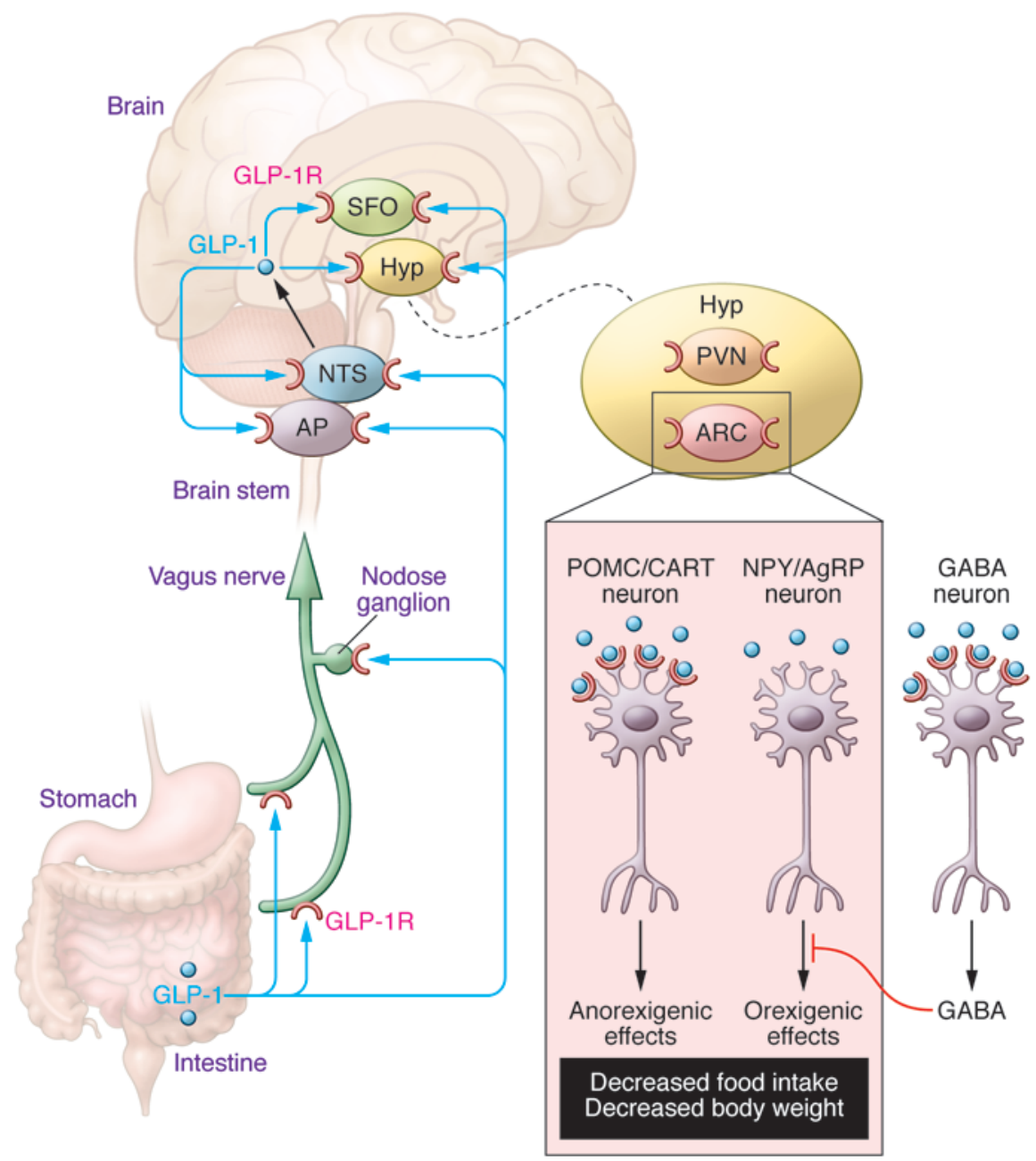

vagus nerve, nodose ganglia, hypothalamic nuclei, and the brain stem. Brain-derived GLP-1 is synthesized in a subset of neurons in the NTS that project to GLP-1R-expressing regions in the hindbrain and hypothalamus, including the paraventricular nucleus (PVN), dorsal medial nucleus of the hypothalamus, and arcuate nucleus (ARC), as well as the NTS itself. A small amount of GLP-1 may also be synthesized within the hypothalamus itself (11), and gut-derived GLP-1 may communicate with the brain by accessing GLP-1Rs within fibers innervating the portal vein or the nodose ganglion of the abdominal vagus nerve. Hence, the relative functional importance of GLP-1Rs in these different sites for the transduction of signals that reduce food intake or body weight is unclear.

\section{Homing in on the CNS}

Two new studies advance our understanding of these concepts, using complementary techniques and approaches. To determine ing region-specific injections of GLP-1R antagonists, lesioning specific nerves and
Figure 1. Peripherally administered GLP-1R agonists reduce food intake and body weight through signaling mechanisms requiring functional GLP-1Rs in the ARC of the hypothalamus. Several regions of the brain express GLP$1 R$ s, including the ARC, PVN, and subfornical organ (SFO), and signals from CLP-1 are transmitted through the vagus nerve or converge on the NTS and area postrema (AP). GLP-1 directly activates POMC/CART neurons and indirectly inhibits, via GABAergic transmission, the neuropeptide Y/agouti-related peptide (NPY/ AgRP) neurons, which collectively results in signals that reduce food intake. Although GLP-1 generates signals that are transmitted through the vagus nerve or converge on the NTS or PVN of the hypothalamus (Hyp), these regions are not required to transduce an anorectic GLP-1Rdependent signal. nuclei, ex vivo electrophysiological studies, and peripheral administration of a fluorescently labeled liraglutide molecule in rats, WT mice, and Glp1r/- mice to dissect mechanisms that link peripheral administration of a GLP-1R agonist to reduced food intake and weight loss. Although liraglutide was taken up by all circumventricular organs as well as the ARC and PVN in the hypothalamus, Secher et al. determined that it is the proopiomelanocortin/cocaine- and amphetamine-regulated transcript (POMC/ CART) neurons in the ARC that are the direct targets of liraglutide-induced weight loss, whereas the NTS, area postrema, PVN, and vagus nerve are not involved. Indeed, GLP-1R blockade by direct injection of exendin(9-39) into the ARC abolished the weight loss effects of liraglutide, whereas neither injection of exendin(9-39) into the PVN nor electrolytic lesion of the PVN affected the ability of liraglutide to reduce weight gain in rats. Remarkably, peripheral administration of fluorescently labeled 
liraglutide led to internalization of the peptide by anorexigenic POMC/CART neurons in the ARC. Secher et al. revealed that the GLP-1R itself is important for mediating the uptake of fluorescently labeled liraglutide into the CNS, as peripheral administration of the peptide failed to accumulate within the CNS of mice with a global knockout of the Glp1r. In contrast, no uptake of peripherally administered liraglutide was detected in the NTS of WT mice. Liraglutide also acted indirectly, via GABAergic neurons, to inhibit the activity of orexigenic neuropeptide Y/agouti-related peptide neurons in the ARC (Figure 1).

The studies from Sisley et al. (12) and Secher et al. (13) indicate that GLP-1Rs on vagal afferent nerves are not required for the pharmacological actions of liraglutide that reduce feeding and body weight in rodents. Intriguingly, Sisley et al. demonstrated that liraglutide still acutely reduces food intake in mice with nestin-Cre-mediated deletion of Glp1r in the CNS, suggesting that maximal GLP-1R-dependent control of short-term food intake may require inputs from GLP-Rs not fully targeted by nestin-Cre expression. Furthermore, the importance of the ARC, but not the PVN, for liraglutide-induced weight loss, as delineated by Secher et al., is in direct contrast to findings from Sandoval et al. (14), who reported that GLP-1Rs in the PVN, but not the ARC, transduce the acute anorectic effects of intracerebral GLP-1 in rats. The discrepancies among the studies from Sisley et al. (12), Secher et al. (13), and others likely reflect differences in experimental paradigms, including the specific GLP-1R agonist used (short acting versus long acting, small molecule versus large molecule, etc.), the route of administration (i.p., s.c., or i.v.), dose, species studied, acute versus chronic analysis, and intrinsic shortcomings in the specific model systems used, such as lack of complete tissue specificity with Cre drivers, incomplete knockdowns, and potential developmental compensation when using constitutively active versus tamoxifen-inducible Cre systems.

\section{Conclusions and future directions}

What are the clinical implications of these findings? First, although some rodent and human studies have implicated a role for increased energy expenditure in GLP-1Rdependent weight loss, the studies by Sisley et al. and Secher et al., together with controlled trials in humans (15), emphasize the dominant role of reduced energy intake for the weight loss effects associated with GLP-1R agonists. The observation that liraglutide is taken up in a GLP-1R-dependent manner and directly binds to neurons within the ARC and other CNS regions raises questions about the central mechanisms of action and the efficacy of larger nonpeptide GLP-1R agonists. Although albiglutide and dulaglutide, two high-molecular-weight GLP$1 \mathrm{R}$ agonists, both produce effective glycemic control in clinical studies, neither of these agents were as effective as liraglutide in generating weight loss in headto-head studies that evaluated maximal doses of drugs approved for the treatment of diabetes $(16,17)$. Whether the greater effectiveness of liraglutide for achieving weight loss reflects enhanced uptake into the CNS, subtle differences in transport or activity of small peptide versus high-molecular-weight GLP-1R agonists at the relevant CNS GLP-1Rs, or simply an incompletely explored dose-response relationship remains unclear. Furthermore, efforts directed at generating combinatorial GLP-1R agonists - with one or two additional peptide agonists to achieve greater weight loss (18) - in a single molecule may need to consider the effect of molecular structure on the GLP$1 \mathrm{R}$-dependent uptake and activity of the hybrid agonists in the CNS. Although the effects of GLP-1R agonists on complex human behaviors linked to reduction in food intake are likely more complex and involve multiple brain regions important for food selection, reward, and appetite $(19,20)$, the rodent studies described by Sisley et al. and Secher et al. will refine our understanding of how peripherally administered GLP-1R agonists control body weight. The successful translation of GLP-1 science from bench to bedside reflects a body of work that is highly reproducible and based on mechanistic studies, which inform the design and rationale use of GLP-1-based therapies. The studies by Sisley et al. and Secher et al. provide important new information that further illuminates pathways and strategies to optimize the use of new GLP-1R agonists for the treatment of human subjects with diabetes and/or obesity.

\section{Acknowledgments}

D.J. Drucker holds a Canada Research Chair in Regulatory Peptides and a Banting and Best Diabetes Centre Novo Nordisk Chair in Incretin Biology and is supported in part by Canadian Institutes for Health Research grant MOP 123391.

Address correspondence to: Daniel J. Drucker, Lunenfeld-Tanenbaum Research Institute, Mt. Sinai Hospital, 600 University Ave., Toronto, Ontario, Canada M5G1X5. Phone: 416.361.2661; E-mail: drucker@lunenfeld.ca.

1. Campbell JE, Drucker DJ. Pharmacology physiology and mechanisms of incretin hormone action. Cell Metab. 2013;17(6):819-837.

2. Meeran K, et al. Repeated intracerebroventricular administration of glucagon-like peptide-1-(7-36) amide or exendin-(9-39) alters body weight in the rat. Endocrinology. 1999;140(1):244-250.

3. Barrera JG, Sandoval DA, D'Alessio DA, Seeley RJ. GLP-1 and energy balance: an integrated model of short-term and long-term control. Nat Rev Endocrinol. 2011;7(9):507-516.

4. Patterson JT, et al. A novel human-based receptor antagonist of sustained action reveals body weight control by endogenous GLP-1. ACS Chem Biol. 2011;6(2):135-145.

5. Steinert RE, et al. Effect of glucagon-like peptide-1 receptor antagonism on appetite and food intake in healthy men. Am J Clin Nutr. 2014;100(2):514-523.

6. Melhorn SJ, Tyagi V, Smeraglio A, Roth CL, Schur EA. Initial evidence that GLP-1 receptor blockade fails to suppress postprandial satiety or promote food intake in humans. Appetite. 2014;82C:85-90.

7. Turton MD, et al. A role for glucagon-like peptide- 1 in the central regulation of feeding. Nature. 1996;379(6560):69-72.

8. Lockie SH, et al. Direct control of brown adipose tissue thermogenesis by central nervous system glucagon-like peptide-1 receptor signaling. Diabetes. 2012;61(11):2753-2762.

9. Drucker DJ, et al. Exenatide once weekly versus twice daily for the treatment of type 2 diabetes: a randomised, open-label, non-inferiority study. Lancet. 2008;372(9645):1240-1250.

10. Nauck MA, Kemmeries G, Holst JJ, Meier JJ. Rapid tachyphylaxis of the glucagon-like peptide 1-induced deceleration of gastric emptying in humans. Diabetes. 2011;60(5):1561-1565.

11. Drucker DJ, Asa S. Glucagon gene expression in vertebrate brain. J Biol Chem. 1988;263(27):13475-13478.

12. Sisley S, et al. Neuronal GLP1R mediates liraglutide's anorectic but not glucose-lowering effect. J Clin Invest. 2014;124(6):2456-2463.

13. Secher A, et al. The arcuate nucleus mediates GLP-1 receptor agonist liraglutide-dependent weight loss. 
JClin Invest. 2014;124(10):4473-4488.

14. Sandoval DA, Bagnol D, Woods SC, D’Alessio DA, Seeley RJ. Arcuate glucagon-like peptide 1 receptors regulate glucose homeostasis but not food intake. Diabetes. 2008;57(8):2046-2054.

15. van Can J, et al. Effects of the once-daily GLP-1 analog liraglutide on gastric emptying, glycemic parameters, appetite and energy metabolism in obese, non-diabetic adults. Int JObes (Lond). 2014;38(6):784-793.

16. Pratley RE, et al. Once-weekly albiglutide versus once-daily liraglutide in patients with type 2 diabetes inadequately controlled on oral drugs (HARMONY 7): a randomised, open-label, multicentre, non-inferiority phase 3 study. Lancet Diabetes Endocrinol. 2014;2(4):289-297.

17. Dungan KM, et al. Once-weekly dulaglutide versus once-daily liraglutide in metformin-treated patients with type 2 diabetes (AWARD-6): a randomised, open-label, phase 3, non-inferiority trial. Lancet. 2014;S0140-6736(14)60976-4.

18. Sadry SA, Drucker DJ. Emerging combina- torial hormone therapies for the treatment of obesity and T2DM. Nat Rev Endocrinol. 2013;9(7):425-433

19. De Silva A, et al. The gut hormones PYY 3-36 and GLP-1 7-36 amide reduce food intake and modulate brain activity in appetite centers in humans. Cell Metab. 2011;14(5):700-706.

20. van Bloemendaal L, et al. GLP-1 receptor activation modulates appetite- and reward-related brain areas in humans [published online ahead of print July 28, 2014]. Diabetes. doi:10.2337/db14-0849. 